Georgian Mathematical Journal

Volume 8 (2001), Number 4, 767-784

\title{
SOME PROBLEMS OF THERMOELASTIC EQUILIBRIUM OF A RECTANGULAR PARALLELEPIPED IN TERMS OF ASYMMETRIC ELASTICITY
}

\author{
N. KHOMASURIDZE
}

\begin{abstract}
An effective solution of a number of boundary value and boundary contact problems of thermoelastic equilibrium is constructed for a homogeneous isotropic rectangular parallelepiped in terms of asymmetric and pseudo-asymmetric elasticity (Cosserat's continuum and pseudo- continuum). Two opposite faces of a parallelepiped are affected by arbitrary surface disturbances and a stationary thermal field, while for the four remaining faces symmetry or anti-symmetry conditions (for a multilayer rectangular parallelepiped nonhomogeneous contact conditions are also defined) are given. The solutions are constructed in trigonometric series using the method of separation of variables.
\end{abstract}

2000 Mathematics Subject Classification: 74B99.

Key words and phrases: Asymptotic thermoelasticity, Cosserat's continuum, Cosserat's pseudo-continuum, boundary value problem, Fourier method, Fourier series.

\section{INTRODUCTION}

There are a number of papers devoted to problems of elastic equilibrium in terms of asymmetric elasticity. Asymmetric elasticity was first introduced by the Cosserat brothers [1] and was later on developed by Truesdell and Toupin [2]. Papers by Kuvshinsky, Aero, Palmov, Grioli, Mindlin, Tiersten and Koiter are devoted to linear theory for Cosserat's medium. These and some other papers are mentioned in [3]. Works by Eringen [4], Nowacki [5], Kupradze, Gegelia, Basheleishvili and Burchuladze [6], Carbonari and Russo [7] are also worth mentioning. Linear theory of asymmetric thermoelasticity was developed by Nowacki [5].

As far as we know, there have been no papers dealing with thermoelastic equilibrium of a rectangular parallelepiped in terms of asymmetric elasticity. This issue is the subject of the given work, in which using the general solution introduced by the author as well as the method of separation of variables and double series, the solutions of some boundary value and boundary contact problems of asymmetric thermoelasticity are constructed for a rectangular parallelepiped occupying the domain $\Omega=\left\{0<x_{1}<x_{11}, 0<x_{2}<x_{21}, 0<x_{3}<x_{31}\right\}$. For the lateral faces $x_{1}=0, x_{1}=x_{11}, x_{2}=0, x_{2}=x_{21}$ symmetry and antisym- 
metry conditions are defined while on the faces $x_{3}=0$ and $x_{3}=x_{31}$ arbitrary surface and thermal disturbances can be applied. In the case of the rectangular parallelepiped which is multilayer along the coordinate $x_{3}$ we have symmetry and antisymmetry conditions again with $x_{1}=0, x_{1}=x_{11}, x_{2}=0, x_{2}=x_{21}$, on the contact surfaces $x_{3}=$ const nonhomogeneous contact conditions are defined while on the two remaining faces arbitrary surface and thermal disturbances can be applied.

1. If no mass forces are applied, the equilibrium equations for a homogeneous isotropic body in the case of Cosserat's continuum have the following form [6]:

$$
\begin{aligned}
& \text { a) } \sum_{j} \frac{\partial N_{j i}}{\partial x_{j}}=0 \\
& \text { b) } \sum_{j} \frac{\partial M_{j i}}{\partial x_{j}}+\sum_{k, j} \varepsilon_{i j k} N_{j k}=0 \\
& \text { c) } \Delta T=0
\end{aligned}
$$

where $i=1,2,3 ; j=1,2,3 ; k=1,2,3 ; \varepsilon_{i j k}$ is Levy-Civita symbol; $x_{1}, x_{2}, x_{3}$ are Cartesian coordinates, $N_{11}, N_{22}, N_{33}$ are normal stresses, $N_{21}, N_{31}, N_{12}, \ldots$, $N_{23}$ are tangential stresses, $M_{11}, M_{22}, M_{33}$ are torsion micromoments; $M_{21}, M_{31}$, $M_{12}, \ldots, M_{23}$ are bending micromoments; $T$ is the change in the temperature of the body; $\Delta=\frac{\partial}{\partial x_{1}^{2}}+\frac{\partial}{\partial x_{2}^{2}}+\frac{\partial}{\partial x_{3}^{2}}$.

The equalities connecting stresses and micromoments with the components of displacement and rotation vectors have the following form [6]

$$
\begin{aligned}
N_{i j} & =\delta_{i j}[\lambda \operatorname{div} \vec{U}-(3 \lambda+2 \mu) \gamma T]+\left(\mu+\sigma_{1}\right) \frac{\partial u_{j}}{\partial x_{i}} \\
& +\left(\mu-\sigma_{1}\right) \frac{\partial u_{i}}{\partial x_{j}}-2 \sigma_{1} \sum_{k} \varepsilon_{i j k} \omega_{k} \\
M_{i j} & =\delta_{i j} \sigma_{2} \operatorname{div} \vec{\omega}+\left(\sigma_{3}+\sigma_{4}\right) \frac{\partial \omega_{j}}{\partial x_{i}}+\left(\sigma_{3}-\sigma_{4}\right) \frac{\partial \omega_{i}}{\partial x_{j}}
\end{aligned}
$$

where $\vec{U}=u_{1} \overrightarrow{l_{1}}+u_{2} \overrightarrow{l_{2}}+u_{3} \overrightarrow{l_{3}}\left(\overrightarrow{l_{1}}, \overrightarrow{l_{2}}, \overrightarrow{l_{3}}\right.$ are basis vectors in the Cartesian coordinate system), $\vec{\omega}=\omega_{1} \overrightarrow{l_{1}}+\omega_{2} \overrightarrow{l_{2}}+\omega_{3} \overrightarrow{l_{3}}$ are displacement and rotation vectors, respectively, $\delta_{i j}$ is Kronecker's symbol, $\lambda, \mu$ are classical elasticity characteristics and $\sigma_{1}, \sigma_{2}, \sigma_{3}, \sigma_{4}$ are asymmetric elasticity characteristics with $\mu>0$, $3 \lambda+2 \mu>0, \sigma_{1}>0, \sigma_{3}>0,3 \sigma_{2}+2 \sigma_{3}>0, \sigma_{4}>0 ; \lambda=\frac{\nu E}{(1-2 \nu)(1+\nu)}, \mu=\frac{E}{2(1-\nu)}$, where $E$ is elasticity modulus, $\nu$ is the Poisson coefficient, $\gamma$ is the coefficient of linear thermal expansion.

Substituting (2) into (1)a, (1)b we have

$$
\begin{aligned}
& \operatorname{grad}[(\lambda+2 \mu) \operatorname{div} \vec{U}-(3 \lambda+2 \mu) \gamma T]-\operatorname{rot}\left[\left(\mu+\sigma_{1}\right) \operatorname{rot} \vec{U}-2 \sigma_{1} \vec{\omega}\right]=0, \\
& \operatorname{grad}\left[\left(\sigma_{2}+2 \sigma_{3}\right) \operatorname{div} \vec{\omega}\right]-\operatorname{rot}\left[\left(\sigma_{3}+\sigma_{4}\right) \operatorname{rot} \vec{\omega}-2 \sigma_{1} \vec{U}\right]-4 \sigma_{1} \vec{\omega}=0 .
\end{aligned}
$$


The second equation of system (3) can be written as

$\operatorname{grad}\left[\left(\sigma_{2}+2 \sigma_{3}\right) \operatorname{div} \vec{\omega}\right]-\operatorname{rot}\left[\left(\sigma_{3}+\sigma_{4}\right) \operatorname{rot} \vec{\omega}+2 \mu \vec{U}\right]+2\left[\left(\mu+\sigma_{1}\right) \operatorname{rot} \vec{U}-2 \sigma_{1} \vec{\omega}\right]=0$

Taking into consideration the last equality we can write

a) $\operatorname{grad}[(\lambda+2 \mu) \operatorname{div} \vec{U}-(3 \lambda+2 \mu) \gamma T]$

$-\operatorname{rot}\left[\left(\mu+\sigma_{1}\right) \operatorname{rot} \vec{U}-2 \sigma_{1} \vec{\omega}\right]=0$,

b) $\operatorname{div}\left[\left(\mu+\sigma_{1}\right) \operatorname{rot} \vec{U}-2 \sigma_{1} \vec{\omega}\right]=-\frac{2 \sigma_{1}}{\sigma_{2}+2 \sigma_{3}}\left[\left(\sigma_{2}+2 \sigma_{3}\right) \operatorname{div} \vec{\omega}\right]$,

c) $\operatorname{grad}\left[\left(\sigma_{2}+2 \sigma_{3}\right) \operatorname{div} \vec{\omega}\right]-\operatorname{rot}\left[\left(\sigma_{3}+\sigma_{4}\right) \operatorname{rot} \vec{\omega}+2 \mu \vec{U}\right]$

$+2\left[\left(\mu+\sigma_{1}\right) \operatorname{rot} \vec{U}-2 \sigma_{1} \vec{\omega}\right]=0$,

d) $\operatorname{div}\left[\left(\sigma_{3}+\sigma_{4}\right) \operatorname{rot} \vec{\omega}+2 \mu \vec{U}\right]=\frac{2 \mu}{\lambda+2 \mu}[(\lambda+2 \mu) \operatorname{div} \vec{U}$

$-(3 \lambda+2 \mu) \gamma T]+\frac{2 \mu(3 \lambda+2 \mu)}{\lambda+2 \mu} \gamma T=0$.

It can be easily seen that (4b) and (4d) are identities.

Without loss of generality we can represent the function $T$ as

$$
T=\frac{2(\lambda+2 \mu)}{(3 \lambda+2 \mu) \gamma} \frac{\partial \widetilde{T}}{\partial x_{3}}
$$

where $\Delta \widetilde{T}=0$. No loss of generality will occur if $T$ is expressed by a derivative of the harmonic function $\widetilde{T}$ since it is preserved by an appropriate representation of the harmonic function $\widetilde{T}$ using the method of separation of variables as in [8].

Introduce the notation
a) $(\lambda+2 \mu) \operatorname{div} \vec{U}-(3 \lambda+2 \mu) \gamma T=D$,
b) $\left(\sigma_{2}+2 \sigma_{3}\right) \operatorname{div} \vec{\omega}=D^{*}$,
c) $\left(\mu+\sigma_{1}\right) \operatorname{rot} \vec{U}-2 \sigma_{1} \vec{\omega}=\vec{K}=K_{1} \overrightarrow{l_{1}}+K_{2} \overrightarrow{l_{2}}+K_{3} \overrightarrow{l_{3}}$,
d) $\left(\sigma_{3}+\sigma_{4}\right) \operatorname{rot} \vec{\omega}+2 \mu \vec{U}=\vec{K}^{*}=K_{1}^{*} \vec{l}_{1}+K_{2}^{*} \vec{l}_{2}+K_{3}^{*} \vec{l}_{3}$.

Taking (5) into account, we can write system (4) in the following way

$$
\begin{array}{ll}
\operatorname{grad} D-\operatorname{rot} \vec{K}=0, & \operatorname{div} \vec{K}=-\frac{2 \sigma_{1}}{\sigma_{2}+2 \sigma_{3}} D^{*}, \\
\operatorname{grad} D^{*}-\operatorname{rot} \vec{K}^{*}+2 \vec{K}=0, & \operatorname{div} \vec{K}^{*}=\frac{2 \mu}{\lambda+2 \mu} D+4 \mu \frac{\partial \widetilde{T}}{\partial x_{3}} .
\end{array}
$$


Finally we obtain the following system
a) $\frac{\partial D}{\partial x_{1}}-\frac{\partial K_{3}}{\partial x_{2}}+\frac{\partial K_{2}}{\partial x_{3}}=0$,
b) $\frac{\partial D}{\partial x_{2}}-\frac{\partial K_{1}}{\partial x_{3}}+\frac{\partial K_{3}}{\partial x_{1}}=0$,
c) $\frac{\partial D}{\partial x_{3}}-\frac{\partial K_{2}}{\partial x_{1}}+\frac{\partial K_{1}}{\partial x_{2}}=0$,
d) $\frac{\partial K_{1}}{\partial x_{1}}+\frac{\partial K_{2}}{\partial x_{2}}+\frac{\partial K_{3}}{\partial x_{3}}=-\frac{2 \sigma_{1}}{\sigma_{2}+2 \sigma_{3}} D^{*}$,
e) $\frac{\partial D^{*}}{\partial x_{1}}-\frac{\partial K_{3}^{*}}{\partial x_{2}}+\frac{\partial K_{2}^{*}}{\partial x_{3}}+2 K_{1}=0$,
f) $\frac{\partial D^{*}}{\partial x_{2}}-\frac{\partial K_{1}^{*}}{\partial x_{3}}+\frac{\partial K_{3}^{*}}{\partial x_{1}}+2 K_{2}=0$,
g) $\frac{\partial D^{*}}{\partial x_{3}}-\frac{\partial K_{2}^{*}}{\partial x_{1}}+\frac{\partial K_{1}^{*}}{\partial x_{2}}+2 K_{3}=0$,
h) $\frac{\partial K_{1}^{*}}{\partial x_{1}}+\frac{\partial K_{2}^{*}}{\partial x_{2}}+\frac{\partial K_{3}^{*}}{\partial x_{3}}=\frac{2 \mu}{\lambda+2 \mu} D+4 \mu \frac{\partial \widetilde{T}}{\partial x_{3}}$;
a) $\frac{\partial u_{1}}{\partial x_{1}}+\frac{\partial u_{2}}{\partial x_{2}}+\frac{\partial u_{3}}{\partial x_{3}}=\frac{D}{\lambda+2 \mu}+2 \frac{\partial \widetilde{T}}{\partial x_{3}}$,
b) $\left(\mu+\sigma_{1}\right)\left(\frac{\partial u_{3}}{\partial x_{2}}-\frac{\partial u_{2}}{\partial x_{3}}\right)-2 \sigma_{1} \omega_{1}=K_{1}$,
c) $\left(\mu+\sigma_{1}\right)\left(\frac{\partial u_{1}}{\partial x_{3}}-\frac{\partial u_{3}}{\partial x_{1}}\right)-2 \sigma_{1} \omega_{2}=K_{2}$,
d) $\left(\mu+\sigma_{1}\right)\left(\frac{\partial u_{2}}{\partial x_{1}}-\frac{\partial u_{1}}{\partial x_{2}}\right)-2 \sigma_{1} \omega_{3}=K_{3}$
e) $\frac{\partial \omega_{1}}{\partial x_{1}}+\frac{\partial \omega_{2}}{\partial x_{2}}+\frac{\partial \omega_{3}}{\partial x_{3}}=\frac{D^{*}}{\sigma_{2}+2 \sigma_{3}}$
f) $\left(\sigma_{3}+\sigma_{4}\right)\left(\frac{\partial \omega_{3}}{\partial x_{2}}-\frac{\partial \omega_{2}}{\partial x_{3}}\right)+2 \mu u_{1}=K_{1}^{*}$,
g) $\left(\sigma_{3}+\sigma_{4}\right)\left(\frac{\partial \omega_{1}}{\partial x_{3}}-\frac{\partial \omega_{3}}{\partial x_{1}}\right)+2 \mu u_{2}=K_{2}^{*}$
h) $\left(\sigma_{3}+\sigma_{4}\right)\left(\frac{\partial \omega_{2}}{\partial x_{1}}-\frac{\partial \omega_{1}}{\partial x_{2}}\right)+2 \mu u_{3}=K_{3}^{*}$,

in 14 unknowns, in particular, the unknowns $D, K_{1}, K_{2}, K_{3}, D^{*}, K_{1}^{*}, K_{2}^{*}, K_{3}^{*}$, $\omega_{1}, \omega_{2}, \omega_{3}, u_{1}, u_{2}, u_{3}$ (the temperature $T$ is defined by (1c) under appropriate boundary conditions). Note that identities (8d) and (8h) complement six equilibrium equations (8a,b,c,e,f,g) to obtain two similar systems (8a,b,c,d) and $(9 \mathrm{e}, \mathrm{f}, \mathrm{g}, \mathrm{h})$ which consist of four differential equations with the first order partial derivatives in four unknowns $D, K_{1}, K_{2}, K_{3}$ and $D^{*}, K_{1}^{*}, K_{2}^{*}, K_{3}^{*}$, respectively (in this case the functions $D^{*}$ and $D$, which also appear in the right-hand side of 
(8d) of system $(8 \mathrm{a}, \mathrm{b}, \mathrm{c}, \mathrm{d})$ and in the right-hand side of $(8 \mathrm{~h})$ of system $(8 \mathrm{c}, \mathrm{f}, \mathrm{g}, \mathrm{h})$, are assumed to be known).

The given work deals with the thermoelastic equilibrium of a rectangular parallelepiped (RP) occupying the domain $\Omega=\left\{0<x_{1}<x_{11}, 0<x_{2}<x_{21}\right.$, $\left.0<x_{3}<x_{31}\right\}$. The boundary conditions appearing in the paper and defining the class of the boundary value problems under consideration are given below.

$$
\text { For } x_{1}=x_{1 s}: \quad \text { a) } \begin{aligned}
u_{1}=0, & N_{12}=0, N_{13}=0, M_{11}=0, \\
\omega_{2} & =0, \omega_{3}=0, \frac{\partial T}{\partial x_{1}}=0 \Longleftrightarrow \\
u_{1}=0, \frac{\partial u_{2}}{\partial x_{1}} & =0, \frac{\partial u_{3}}{\partial x_{1}}=0, \frac{\partial \omega_{1}}{\partial x_{1}}=0, \\
\omega_{2} & =0, \omega_{3}=0, \frac{\partial T}{\partial x_{1}}=0
\end{aligned}
$$

or

b) $N_{11}=0, u_{2}=0, u_{3}=0, \omega_{1}=0 M_{12}=0$,

$$
\begin{gathered}
M_{13}=0, T=0 \Longleftrightarrow \\
\frac{\partial u_{1}}{\partial x_{1}}=0, u_{2}=0, u_{3}=0, \omega_{1}=0, \frac{\partial \omega_{2}}{\partial x_{1}}=0, \\
\frac{\partial \omega_{3}}{\partial x_{1}}=0, T=0 .
\end{gathered}
$$

$$
\text { For } x_{2}=x_{2 s}: \quad \text { a) } \begin{aligned}
u_{2}=0, & N_{23}=0, N_{21}=0, M_{22}=0, \\
\omega_{3} & =0, \omega_{1}=0, \frac{\partial T}{\partial x_{2}}=0 \Longleftrightarrow \\
& \begin{aligned}
u_{2}=0, \frac{\partial u_{3}}{\partial x_{2}} & =0, \frac{\partial u_{1}}{\partial x_{2}}=0, \frac{\partial \omega_{2}}{\partial x_{2}}=0, \\
\omega_{3} & =0, \omega_{1}=0, \frac{\partial T}{\partial x_{2}}=0
\end{aligned}
\end{aligned}
$$

or

$$
\text { b) } \begin{gathered}
N_{22}=0, u_{3}=0, u_{1}=0, \omega_{2}=0 M_{23}=0, \\
M_{21}=0, T=0 \Longleftrightarrow \\
\frac{\partial u_{2}}{\partial x_{2}}=0, u_{3}=0, u_{1}=0, \omega_{2}=0, \frac{\partial \omega_{3}}{\partial x_{2}}=0, \\
\frac{\partial \omega_{1}}{\partial x_{2}}=0, T=0 .
\end{gathered}
$$

$$
\text { For } x_{3}=x_{3 s}: \quad \begin{aligned}
& g_{s 1} N_{33}+\widetilde{g}_{s 1} \cdot u_{3}=f_{s 1}\left(x_{1}, x_{2}\right), \\
& g_{s 2} N_{31}+\widetilde{g}_{s 2} \cdot u_{1}=f_{s 2}\left(x_{1}, x_{2}\right), \\
& g_{s 3} N_{32}+\widetilde{g}_{s 3} \cdot u_{2}=f_{s 3}\left(x_{1}, x_{2}\right), \\
& g_{s 4} M_{33}+\widetilde{g}_{s 4} \cdot \omega_{3}=f_{s 4}\left(x_{1}, x_{2}\right), \\
& g_{s 5} M_{31}+\widetilde{g}_{s 5} \cdot \omega_{1}=f_{s 5}\left(x_{1}, x_{2}\right), \\
& g_{s 6} M_{32}+\widetilde{g}_{s 6} \cdot \omega_{2}=f_{s 6}\left(x_{1}, x_{2}\right), \\
& g_{s 7} \frac{\partial T}{\partial x_{3}}+\widetilde{g}_{s 7} T=f_{s 7}\left(x_{1}, x_{2}\right) .
\end{aligned}
$$

In (10), (11) and (12) we have $s=0,1$ with $x_{10}=x_{20}=x_{30}=0 ; g_{s 1}, \widetilde{g}_{s 1}, \ldots$, $\widetilde{g}_{s 7}$ being the defined constants governed by the conditions $g_{s 1} \cdot \widetilde{g}_{s 1} \geq 0, g_{s 2} \cdot \widetilde{g}_{s 2} \geq$ $0, \ldots, g_{s 7} \cdot \widetilde{g}_{s 7} \geq 0$. The conditions imposed on the functions $f_{s 1}, f_{s 2}, \ldots, f_{s 7}$ will be described in below, we can just note that the functions are taken so that on the edges of the RP the compatibility conditions are satisfied. With $\widetilde{g}_{s 1}=$ $\widetilde{g}_{s 2}=\widetilde{g}_{s 3}=0, \widetilde{g}_{s 4}=\widetilde{g}_{s 5}=\widetilde{g}_{s 6}=0$ we have the boundary conditions for the first problem of asymmetric thermoelasticity, with $g_{s 1}=g_{s 2}=g_{s 3}=0, g_{s 4}=g_{s 5}=$ $g_{s 6}=0$ we have the boundary conditions for the second problem of asymmetric thermoelasticity, etc. Note that (12) implies that with $x_{3}=0$ one type of 
conditions can be defined while with $x_{3}=x_{31}$ there can be another conditions. Conditions (10a) and (11a) are called the symmetry conditions, while conditions (10b) and (11b) are called the antisymmetry conditions. We should also note that conditions (10) and (11) are the conditions of the continuous extension of the solution across the corresponding face of the RP. The validity of this statements is a direct consequence of the constructed solutions of the boundary value problems. It is interesting to observe that the technical interpretation of boundary conditions (10) and (11) is the same as of the corresponding conditions in $[3]$.

Theorem 1. The general solution of system (7), (6) or, what is the same, system (8), (9) according to asymmetric theory has the following form

$$
\begin{gathered}
\omega_{1}=\frac{\partial \psi_{3}}{\partial x_{1}}+\frac{\varkappa}{2} \frac{\partial \varphi_{1}}{\partial x_{2}}+\frac{1}{2} \frac{\partial^{2} \varphi_{2}}{\partial x_{1} \partial x_{3}}+\frac{\partial \psi_{1}}{\partial x_{3}}, \\
\omega_{2}=\frac{\partial \psi_{3}}{\partial x_{2}}-\frac{\varkappa}{2} \frac{\partial \varphi_{1}}{\partial x_{1}}+\frac{1}{2} \frac{\partial^{2} \varphi_{2}}{\partial x_{2} \partial x_{3}}+\frac{\partial \psi_{2}}{\partial x_{3}}, \\
\omega_{3}=\frac{\partial \psi_{3}}{\partial x_{3}}+\frac{1}{2} \frac{\partial^{2} \varphi_{2}}{\partial x_{3}^{2}}-\frac{\partial \psi_{1}}{\partial x_{1}}-\frac{\partial \psi_{2}}{\partial x_{2}} ; \\
u_{1}=\frac{\partial}{\partial x_{1}}\left(\varphi_{3}-x_{3} \varphi_{1}\right)+\frac{\partial \varphi_{2}}{\partial x_{2}} \\
-\frac{\sigma_{3}+\sigma_{4}}{2 \mu}\left(\frac{\partial^{2} \psi_{2}}{\partial x_{1}^{2}}-q \psi_{2}-\frac{\partial^{2} \psi_{1}}{\partial x_{1} \partial x_{2}}\right)+x_{3} \frac{\partial \widetilde{T}}{\partial x_{1}} \\
u_{2}=\frac{\partial}{\partial x_{2}}\left(\varphi_{3}-x_{3} \varphi_{1}\right)-\frac{\partial \varphi_{2}}{\partial x_{1}} \\
+\frac{\sigma_{3}+\sigma_{4}}{2 \mu}\left(\frac{\partial^{2} \psi_{1}}{\partial x_{2}^{2}}-q \psi_{1}-\frac{\partial^{2} \psi_{2}}{\partial x_{1} \partial x_{2}}\right)+x_{3} \frac{\partial \widetilde{T}}{\partial x_{2}}, \\
u_{3}=\frac{\partial}{\partial x_{3}}\left(\varphi_{3}-x_{3} \varphi_{1}\right)+\varkappa \varphi_{1} \\
-\frac{\sigma_{3}+\sigma_{4}}{2 \mu}\left(\frac{\partial^{2} \psi_{2}}{\partial x_{1} \partial x_{3}}-\frac{\partial^{2} \psi_{1}}{\partial x_{2} \partial x_{3}}\right)+x_{3} \frac{\partial \widetilde{T}}{\partial x_{3}}+\widetilde{T} .
\end{gathered}
$$

In (13) and (14) $\varphi_{1}, \varphi_{2}, \varphi_{3}$ and $\widetilde{T}$ are harmonic functions, $\psi_{1}, \psi_{2}, \psi_{3}$ are metaharmonic functions with $\Delta \psi_{1}-q \psi_{1}=0, \Delta \psi_{2}-q \psi_{2}=0, \Delta \psi_{3}-\frac{4 \sigma_{1}}{\sigma_{2}+2 \sigma_{3}} \psi_{3}=0$ and $q=\frac{4 \mu \sigma_{1}}{\left(\mu+\sigma_{1}\right)\left(\sigma_{3}+\sigma_{4}\right)}$.

Proof. (8a, b, c) implies

$$
\Delta D=0
$$


Without loss of generality assume

$$
D=\varkappa \mu \frac{\partial \varphi_{1}}{\partial x_{3}}
$$

where $\Delta \varphi_{1}=0$ (see the comments to $\left.(5)\right)$ and $\varkappa=\frac{2(\lambda+2 \mu)}{\lambda+\mu}=4(1-\nu)$, so taking (15) into account we can write $(8 \mathrm{a}, \mathrm{b}, \mathrm{c}, \mathrm{d})$ as
a) $\frac{\partial}{\partial x_{3}}\left(K_{2}+\varkappa \mu \frac{\partial \varphi_{1}}{\partial x_{1}}\right)-\frac{\partial K_{3}}{\partial x_{2}}=0$,
b) $\frac{\partial K_{3}}{\partial x_{1}}-\frac{\partial}{\partial x_{3}}\left(K_{1}-\varkappa \mu \frac{\partial \varphi_{1}}{\partial x_{2}}\right)=0$,
c) $\frac{\partial}{\partial x_{2}}\left(K_{1}-\varkappa \mu \frac{\partial \varphi_{1}}{\partial x_{2}}\right)-\frac{\partial}{\partial x_{1}}\left(K_{2}+\varkappa \mu \frac{\partial \varphi_{1}}{\partial x_{1}}\right)=0$,
d) $\frac{\partial K_{1}}{\partial x_{1}}+\frac{\partial K_{2}}{\partial x_{2}}+\frac{\partial K_{3}}{\partial x_{3}}=-\frac{2 \sigma_{1}}{\sigma_{2}+2 \sigma_{3}} D^{*}$.

From (16a, b, c) we have

$$
K_{1}=\frac{\partial \widetilde{\varphi}_{2}}{\partial x_{1}}+\varkappa \mu \frac{\partial \varphi_{1}}{\partial x_{2}}, K_{2}=\frac{\partial \widetilde{\varphi}_{2}}{\partial x_{2}}-\varkappa \mu \frac{\partial \varphi_{1}}{\partial x_{1}}, K_{3}=\frac{\partial \widetilde{\varphi}_{2}}{\partial x_{3}} .
$$

Substituting (18) into (16d) we obtain

$$
\Delta \tilde{\varphi}_{2}=-\frac{2 \sigma_{1}}{\sigma_{2}+2 \sigma_{3}} D^{*}
$$

(8e, f, g) implies

$$
\Delta D^{*}-\frac{4 \sigma_{1}}{\sigma_{2}+2 \sigma_{3}} D^{*}=0 .
$$

If we write the solution of this equation as

$$
D^{*}=4 \sigma_{1} \psi_{3},
$$

where $\Delta \psi_{3}-\frac{4 \sigma_{1}}{\sigma_{2}+2 \sigma_{3}} \psi_{3}=0$, then the solution of (18) can be represented as

$$
\widetilde{\varphi}_{2}=\mu \frac{\partial \varphi_{2}}{\partial x_{3}}-2 \sigma_{1} \cdot \psi_{3}
$$

where $\Delta \varphi_{2}=0$ (see the comments to (5)). Taking (19) into account we can write (17) as

$$
\begin{aligned}
& K_{1}=\mu \frac{\partial^{2} \varphi_{2}}{\partial x_{1} \partial x_{3}}-2 \sigma_{1} \frac{\partial \psi_{3}}{\partial x_{1}}+\varkappa \mu \frac{\partial \varphi_{1}}{\partial x_{2}} \\
& K_{2}=\mu \frac{\partial^{2} \varphi_{2}}{\partial x_{2} \partial x_{3}}-2 \sigma_{1} \frac{\partial \psi_{3}}{\partial x_{2}}-\varkappa \mu \frac{\partial \varphi_{1}}{\partial x_{1}} \\
& K_{3}=\mu \frac{\partial^{2} \varphi_{2}}{\partial x_{3}^{2}}-2 \sigma_{1} \frac{\partial \psi_{3}}{\partial x_{3}}
\end{aligned}
$$


With (20) in mind (8e, f, g, h) can be written as
a) $\frac{\partial}{\partial x_{3}}\left(K_{2}^{*}+2 \mu \frac{\partial \varphi_{2}}{\partial x_{1}}\right)-\frac{\partial}{\partial x_{2}}\left(K_{3}^{*}-2 \varkappa \mu \varphi_{1}\right)=0$,
b) $\frac{\partial}{\partial x_{1}}\left(K_{3}^{*}-2 \varkappa \mu \varphi_{1}\right)-\frac{\partial}{\partial x_{3}}\left(K_{1}^{*}-2 \mu \frac{\partial \varphi_{2}}{\partial x_{2}}\right)=0$,
c) $\frac{\partial}{\partial x_{2}}\left(K_{1}^{*}-2 \mu \frac{\partial \varphi_{2}}{\partial x_{2}}\right)-\frac{\partial}{\partial x_{1}}\left(K_{2}^{*}+2 \mu \frac{\partial \varphi_{2}}{\partial x_{1}}\right)=0$,
d) $\frac{\partial K_{1}^{*}}{\partial x_{1}}+\frac{\partial K_{2}^{*}}{\partial x_{2}}+\frac{\partial K_{3}^{*}}{\partial x_{3}}=\frac{2 \varkappa \mu^{2}}{\lambda+2 \mu} \frac{\partial \varphi_{1}}{\partial x_{3}}+4 \mu \frac{\partial \widetilde{T}}{\partial x_{3}}$.

From $(21 \mathrm{a}, \mathrm{b}, \mathrm{c})$ we have

$$
K_{1}^{*}=\frac{\partial \widetilde{\varphi}_{3}}{\partial x_{1}}+2 \mu \frac{\partial \varphi_{2}}{\partial x_{2}}, \quad K_{2}^{*}=\frac{\partial \widetilde{\varphi}_{3}}{\partial x_{2}}-2 \mu \frac{\partial \varphi_{2}}{\partial x_{1}}, \quad K_{3}^{*}=\frac{\partial \widetilde{\varphi}_{3}}{\partial x_{3}}+2 \varkappa \mu \varphi_{1}
$$

Substituting (22) into (21d) we have

$$
\Delta \widetilde{\varphi}_{3}=4 \mu \frac{\partial}{\partial x_{3}}\left(\widetilde{T}-\varphi_{1}\right) .
$$

The solution of this equations is

$$
\widetilde{\varphi}_{3}=2 \mu \varphi_{3}^{*}-2 \mu x_{3}\left(\varphi_{1}-\widetilde{T}\right)
$$

where $\Delta \varphi_{3}^{*}=0$.. If we substitute (23) into (22) then

$$
\begin{aligned}
& K_{1}^{*}=2 \mu \frac{\partial \varphi_{3}^{*}}{\partial x_{1}}-2 \mu x_{3} \frac{\partial \varphi_{1}}{\partial x_{1}}+2 \mu \frac{\partial \varphi_{2}}{\partial x_{2}}+2 \mu x_{3} \frac{\partial \widetilde{T}}{\partial x_{1}}, \\
& K_{2}^{*}=2 \mu \frac{\partial \varphi_{3}^{*}}{\partial x_{2}}-2 \mu x_{3} \frac{\partial \varphi_{1}}{\partial x_{2}}-2 \mu \frac{\partial \varphi_{2}}{\partial x_{1}}+2 \mu x_{3} \frac{\partial \widetilde{T}}{\partial x_{2}}, \\
& K_{3}^{*}=2 \mu \frac{\partial \varphi_{3}^{*}}{\partial x_{3}}-2 \mu x_{3} \frac{\partial \varphi_{1}}{\partial x_{3}}+2 \mu x_{3} \frac{\partial \widetilde{T}}{\partial x_{3}}+2 \mu(4 \varkappa-1) \varphi_{1}+2 \mu \widetilde{T}
\end{aligned}
$$

Applying rot to (6d) and substituting it into (6c) with $\operatorname{rot} \operatorname{rot} \vec{\omega}=\operatorname{grad} \operatorname{div} \vec{\omega}-$ $\Delta \vec{\omega}$, in mind we obtain

$$
\Delta \vec{\omega}-q \vec{\omega}=\frac{1}{\sigma_{2}+2 \sigma_{3}} \operatorname{grad} D^{*}-\frac{1}{\sigma_{3}+\sigma_{4}} \operatorname{rot} \vec{K}^{*}+\frac{q}{2 \sigma_{1}} \vec{K}
$$

If we project this equation onto the coordinate axes and take into account 
equality $D^{*}=4 \sigma_{1} \psi_{3}$ and formulas (20) and (24) we shall have
a) $\Delta \omega_{1}-q \omega_{1}=\left(\frac{4 \sigma_{1}}{\sigma_{2}+2 \sigma_{3}}-q\right) \frac{\partial \psi_{3}}{\partial x_{1}}-\frac{q}{2}\left(\varkappa \frac{\partial \varphi_{1}}{\partial x_{2}}+\frac{\partial^{2} \varphi_{2}}{\partial x_{1} \partial x_{3}}\right)$,
b) $\Delta \omega_{2}-q \omega_{2}=\left(\frac{4 \sigma_{1}}{\sigma_{2}+2 \sigma_{3}}-q\right) \frac{\partial \psi_{3}}{\partial x_{2}}+\frac{q}{2}\left(\varkappa \frac{\partial \varphi_{1}}{\partial x_{1}}-\frac{\partial^{2} \varphi_{2}}{\partial x_{2} \partial x_{3}}\right)$,
c) $\Delta \omega_{3}-q \omega_{3}=\left(\frac{4 \sigma_{1}}{\sigma_{2}+2 \sigma_{3}}-q\right) \frac{\partial \psi_{3}}{\partial x_{3}}-\frac{q}{2} \frac{\partial^{2} \varphi_{2}}{\partial x_{3}^{2}}$.

The solution of (25a) (25b) will be

$$
\begin{aligned}
& \omega_{1}=\frac{\partial \psi_{3}}{\partial x_{1}}+\frac{1}{2}\left(\varkappa \frac{\partial \varphi_{1}}{\partial x_{2}}+\frac{\partial^{2} \varphi_{2}}{\partial x_{1} \partial x_{3}}\right)+\frac{\partial \psi_{1}}{\partial x_{3}}, \\
& \omega_{2}=\frac{\partial \psi_{3}}{\partial x_{2}}-\frac{1}{2}\left(\varkappa \frac{\partial \varphi_{1}}{\partial x_{1}}-\frac{\partial^{2} \varphi_{2}}{\partial x_{2} \partial x_{3}}\right)+\frac{\partial \psi_{2}}{\partial x_{3}},
\end{aligned}
$$

respectively, where $\Delta \psi_{1}-q \psi_{1}=0, \Delta \psi_{2}-q \psi_{2}=0$. We define $\omega_{3}$ from equality $(9 \mathrm{e})$

$$
\omega_{3}=\frac{\partial \psi_{3}}{\partial x_{3}}+\frac{1}{2} \frac{\partial^{2} \varphi_{2}}{\partial x_{3}^{2}}-\frac{\partial \psi_{1}}{\partial x_{1}}-\frac{\partial \psi_{2}}{\partial x_{2}}+f\left(x_{1}, x_{2}\right),
$$

where $f$ is a function resulting from integration of (9e) and satisfying the following equation and the following conditions:

$$
\begin{gathered}
\frac{\partial^{2} f}{\partial x_{1}^{2}}+\frac{\partial^{2} f}{\partial x_{2}^{2}}-q f=0 \\
\frac{\partial f}{\partial x_{s}}=0 \text { or } f=0 \text { with } x_{s}=0, \\
\frac{\partial f}{\partial x_{s}}=0 \text { or } f=0 \text { with } x_{s}=x_{s 1},
\end{gathered}
$$

where $s=1,2$. The solution of $(28)$ is $f=0$.

Substituting (26), (27) and (24) into (9f,g,h) we have

$$
\begin{aligned}
u_{1} & =\frac{\partial}{\partial x_{1}}\left[\varphi_{3}^{*}-\frac{\varkappa\left(\sigma_{3}+\sigma_{4}\right)}{4 \mu} \frac{\partial \varphi_{1}}{\partial x_{3}}\right]-x_{3} \frac{\partial \varphi_{1}}{\partial x_{1}}+\frac{\partial \varphi_{2}}{\partial x_{2}} \\
& -\frac{\sigma_{3}+\sigma_{4}}{2 \mu}\left(\frac{\partial^{2} \psi_{2}}{\partial x_{1}^{2}}-q \psi_{2}-\frac{\partial^{2} \psi_{1}}{\partial x_{1} \partial x_{2}}\right)+x_{3} \frac{\partial \widetilde{T}}{\partial x_{1}}, \\
u_{2} & =\frac{\partial}{\partial x_{2}}\left[\varphi_{3}^{*}-\frac{\varkappa\left(\sigma_{3}+\sigma_{4}\right)}{4 \mu} \frac{\partial \varphi_{1}}{\partial x_{3}}\right]-x_{3} \frac{\partial \varphi_{1}}{\partial x_{2}}-\frac{\partial \varphi_{2}}{\partial x_{1}} \\
& +\frac{\sigma_{3}+\sigma_{4}}{2 \mu}\left(\frac{\partial^{2} \psi_{1}}{\partial x_{2}^{2}}-q \psi_{1}-\frac{\partial^{2} \psi_{2}}{\partial x_{1} \partial x_{2}}\right)+x_{3} \frac{\partial \widetilde{T}}{\partial x_{2}}, \\
u_{3} & =\frac{\partial}{\partial x_{3}}\left[\varphi_{3}^{*}-\frac{\varkappa\left(\sigma_{3}+\sigma_{4}\right)}{4 \mu} \frac{\partial \varphi_{1}}{\partial x_{3}}\right]-x_{3} \frac{\partial \varphi_{1}}{\partial x_{3}}+(\varkappa-1) \varphi_{1}
\end{aligned}
$$




$$
-\frac{\sigma_{3}+\sigma_{4}}{2 \mu}\left(\frac{\partial^{2} \psi_{2}}{\partial x_{1} \partial x_{3}}-\frac{\partial^{2} \psi_{1}}{\partial x_{2} \partial x_{3}}\right)+x_{3} \frac{\partial \widetilde{T}}{\partial x_{3}}+\widetilde{T} .
$$

If we introduce the notation $\left[\varphi_{3}^{*}-\frac{\varkappa\left(\sigma_{3}+\sigma_{4}\right)}{4 \mu} \frac{\partial \varphi_{1}}{\partial x_{3}}\right]=\varphi_{3}$, where, naturally, $\Delta \varphi_{3}=$ 0 , we can say that Theorem 1 is proved.

2. In the case of Cosserat's pseudo-continuum equations (1) still hold, but instead of (2) we have [3]

$$
\begin{aligned}
N_{i j} & =\delta_{i j}[\lambda \operatorname{div} \vec{U}-(3 \lambda+2 \mu) \gamma T]+\mu\left(\frac{\partial u_{j}}{\partial x_{i}}+\frac{\partial u_{i}}{\partial x_{j}}\right) \\
& -\eta_{1} \sum_{k} \varepsilon_{i j k} \Delta\left(\operatorname{rot}_{k} \vec{U}\right), \\
M_{i j} & =2 \eta_{1} \frac{\partial}{\partial x_{i}}\left(\operatorname{rot}_{j} \vec{U}\right)+2 \eta_{2} \frac{\partial}{\partial x_{j}}\left(\operatorname{rot}_{i} \vec{U}\right),
\end{aligned}
$$

where $\eta_{1}, \eta_{2}$ are asymmetric elasticity characteristics with $\eta_{1}>0, \eta_{2}>0$. Substituting (29) into (1) we have

$$
\begin{gathered}
\text { a) } \operatorname{grad}[(\lambda+2 \mu) \operatorname{div} \vec{U}-(3 \lambda+2 \mu) \gamma T] \\
-\operatorname{rot}\left(\mu \operatorname{rot} \vec{U}-\eta_{1} \Delta \operatorname{rot} \vec{U}\right)=0, \\
\text { b) } \operatorname{div}\left(\mu \operatorname{rot} \vec{U}-\eta_{1} \Delta \operatorname{rot} \vec{U}\right)=0,
\end{gathered}
$$

where (30b) is a known identity. Write (30) as

$$
\left\{\begin{array}{l}
\operatorname{grad} D-\operatorname{rot} \vec{K}^{*}=0 \\
\operatorname{div} \vec{K}^{*}=0
\end{array}\right.
$$

where $D=(\lambda+2 \mu) \operatorname{div} \vec{U}-(3 \lambda+2 \mu) \gamma T, \vec{K}^{*}=K_{1}^{*} \vec{l}_{1}+K_{2}^{*} \vec{l}_{2}+K_{3}^{*} \vec{l}_{3}, \vec{K}^{*}=$ $\vec{K}-\frac{\eta_{1}}{\mu} \Delta \vec{K}$ and $\vec{K}=\mu \operatorname{rot} \vec{U}=K_{1} \vec{l}_{1}+K_{2} \vec{l}_{2}+K_{3} \vec{l}_{3}=\mu \operatorname{rot}_{1} \vec{U} \cdot \overrightarrow{l_{1}}+\mu \operatorname{rot}_{2} \vec{U} \cdot$ $\overrightarrow{l_{2}}+\mu \operatorname{rot}_{3} \vec{U} \cdot \vec{l}_{3}$. If we write (31) in a scalar form, we have
a) $\frac{\partial D}{\partial x_{1}}-\frac{\partial K_{3}^{*}}{\partial x_{2}}+\frac{\partial K_{2}^{*}}{\partial x_{3}}=0$
b) $\frac{\partial D}{\partial x_{2}}-\frac{\partial K_{1}^{*}}{\partial x_{3}}+\frac{\partial K_{3}^{*}}{\partial x_{1}}=0$,
c) $\frac{\partial D}{\partial x_{3}}-\frac{\partial K_{2}^{*}}{\partial x_{1}}+\frac{\partial K_{1}^{*}}{\partial x_{2}}=0$
d) $\frac{\partial K_{1}^{*}}{\partial x_{1}}+\frac{\partial K_{2}^{*}}{\partial x_{2}}+\frac{\partial K_{3}^{*}}{\partial x_{3}}=0$.

As in the case of Cosserat's continuum for a RP, we can state boundary conditions defining the class of solvable boundary value problems considered 
according to pseudo-asymmetric thermoelasticity.

$$
\begin{aligned}
& \text { For } x_{1}=x_{1 s}: \quad \text { a) } \quad u_{1}=0, N_{12}=0, N_{13}=0 \text {, } \\
& K_{2}=0, K_{3}=0, \frac{\partial T}{\partial x_{1}}=0 \Longleftrightarrow \\
& u_{1}=0, \frac{\partial u_{2}}{\partial x_{1}}=0, \frac{\partial u_{3}}{\partial x_{1}}=0, \frac{\partial^{2} u_{1}}{\partial x_{1}^{2}}=0, \frac{\partial^{3} u_{2}}{\partial x_{1}^{3}}=0, \\
& \frac{\partial^{3} u_{3}}{\partial x_{1}^{3}}=0, \frac{\partial T}{\partial x_{1}}=0 \\
& \text { b) } N_{11}=0, u_{2}=0, u_{3}=0 \text {, } \\
& M_{12}=0, M_{13}=0, T=0 \Longleftrightarrow \\
& \frac{\partial u_{1}}{\partial x_{1}}=0, u_{2}=0, u_{3}=0, \frac{\partial^{2} u_{2}}{\partial x_{1}^{2}}=0, \frac{\partial^{2} u_{3}}{\partial x_{1}^{2}}=0, \\
& \frac{\partial^{3} u_{1}}{\partial x_{1}^{3}}=0, T=0 \text {. }
\end{aligned}
$$

It should be noted that in (32a) the fourth, the fifth and the sixth conditions, appearing to the right of the equivalence sign, have been obtained assuming that with $x_{1}=x_{1 s}$, besides the defined boundary conditions, equation (32a) also holds. In its turn, in (33b) the sixth condition to the right of the equivalence sign has been obtained assuming that with $x_{1}=x_{1 s}$ equations $(32 \mathrm{a}),(32 \mathrm{~b})$ and (32c) differentiated, correspondingly, with respect to $x_{1}, x_{2}$ and $x_{3}$ are true.

$$
\text { For } x_{2}=x_{2 s}: \quad \text { a) } \begin{aligned}
u_{2}= & 0, N_{23}=0, N_{21}=0, \\
& K_{3}=0, K_{1}=0, \frac{\partial T}{\partial x_{2}}=0 \Longleftrightarrow \\
u_{2}= & 0, \frac{\partial u_{3}}{\partial x_{2}}=0, \frac{\partial u_{1}}{\partial x_{2}}=0, \frac{\partial^{2} u_{2}}{\partial x_{2}^{2}}=0, \frac{\partial^{3} u_{3}}{\partial x_{2}^{3}}=0, \\
& \frac{\partial^{3} u_{1}}{\partial x_{2}^{3}}=0, \frac{\partial T}{\partial x_{2}}=0
\end{aligned}
$$

or

$$
\begin{aligned}
& \text { b) } N_{22}=0, u_{3}=0, u_{1}=0, \\
& M_{21}=0, M_{23}=0, T=0 \Longleftrightarrow \\
& \frac{\partial u_{2}}{\partial x_{2}}=0, u_{3}=0, u_{1}=0, \frac{\partial^{2} u_{3}}{\partial x_{2}^{2}}=0, \frac{\partial^{2} u_{1}}{\partial x_{2}^{2}}=0, \\
& \frac{\partial^{3} u_{2}}{\partial x_{2}^{3}}=0, T=0 .
\end{aligned}
$$

Remarks similar to those conditions (33) take place.

$$
\text { For } x_{3}=x_{3 s}: \quad \begin{aligned}
& g_{s 1} N_{33}+\widetilde{g}_{s 1} u_{3}=f_{s 1}\left(x_{1}, x_{2}\right), \\
& g_{s 2} N_{31}+\widetilde{g}_{s 2} u_{1}=f_{s 2}\left(x_{1}, x_{2}\right), \\
& g_{s 3} N_{32}+\widetilde{g}_{s 3} u_{2}=f_{s 3}\left(x_{1}, x_{2}\right), \\
& g_{s 4} M_{31}+\widetilde{g}_{s 4} K_{1}=f_{s 4}\left(x_{1}, x_{2}\right), \\
& g_{s 5} M_{32}+\widetilde{g}_{s 5} K_{2}=f_{s 5}\left(x_{1}, x_{2}\right), \\
& g_{s 6} \frac{\partial T}{\partial x_{3}}+\widetilde{g}_{s 6} T=f_{s 6}\left(x_{1}, x_{2}\right) .
\end{aligned}
$$

Comments to conditions (12) also hold for conditions (35) and to make it clear we shall point out to following.

In (33), (34) and (35) we have $s=0,1$; (33a) and (34a) are symmetry conditions and (33b) and (34b) are antisymmetry conditions. Conditions (33) and (34) are the conditions of the continuous extension of the solution across the corresponding face of the RP onto a domain which is a mirror reflection with 
respect to this face. The validity of the statement is a direct implication of the constructed solution of the boundary value problems.

Theorem 2. The general solution of system (31) according to pseudo-asymmetric theory has the following form:

$$
\begin{aligned}
u_{1} & =\frac{\partial}{\partial x_{1}}\left(\varphi_{3}-x_{3} \varphi_{1}\right)+\frac{\partial \varphi_{2}}{\partial x_{2}} \\
& -\eta_{1}\left(\eta_{1} \frac{\partial^{2} \psi_{2}}{\partial x_{1}^{2}}-\eta_{1} \frac{\partial^{2} \psi_{1}}{\partial x_{1} \partial x_{2}}+\mu \psi_{2}\right)+x_{3} \frac{\partial \widetilde{T}}{\partial x_{1}} \\
u_{2} & =\frac{\partial}{\partial x_{2}}\left(\varphi_{3}-x_{3} \varphi_{1}\right)-\frac{\partial \varphi_{2}}{\partial x_{1}} \\
& -\eta_{1}\left(\eta_{1} \frac{\partial^{2} \psi_{2}}{\partial x_{1} \partial x_{2}}-\eta_{1} \frac{\partial^{2} \psi_{1}}{\partial x_{2}^{2}}+\mu \psi_{1}\right)+x_{3} \frac{\partial \widetilde{T}}{\partial x_{2}} \\
u_{3} & =\frac{\partial}{\partial x_{3}}\left(\varphi_{3}-x_{3} \varphi_{1}\right)+\varkappa \varphi_{1} \\
& -\eta_{1}^{2}\left(\frac{\partial^{2} \psi_{2}}{\partial x_{1} \partial x_{3}}-\frac{\partial^{2} \psi_{1}}{\partial x_{2} \partial x_{3}}\right)+x_{3} \frac{\partial \widetilde{T}}{\partial x_{3}}+\widetilde{T}
\end{aligned}
$$

In $(36) \varphi_{1}, \varphi_{2}, \varphi_{3}$ and $\widetilde{T}$ are harmonic functions and $\psi_{1}$ and $\psi_{2}$ are metaharmonic functions with $\Delta \psi_{s}-\frac{\mu}{\eta_{1}} \psi_{s}=0$, where $s=1,2$.

Proof. (32a,b,c) implies

$$
\Delta D=0
$$

If we assume

$$
D=\varkappa \mu \frac{\partial \varphi_{1}}{\partial x_{3}}
$$

where $\Delta \varphi_{1}=0$, then taking (37) into account, we can write (32) as

$$
\begin{aligned}
& \frac{\partial}{\partial x_{3}}\left(K_{2}^{*}+\varkappa \mu \frac{\partial \varphi_{1}}{\partial x_{1}}\right)-\frac{\partial K_{3}^{*}}{\partial x_{2}}=0 \\
& \frac{\partial K_{3}^{*}}{\partial x_{1}}-\frac{\partial}{\partial x_{3}}\left(K_{1}^{*}-\varkappa \mu \frac{\partial \varphi_{1}}{\partial x_{2}}\right)=0 \\
& \frac{\partial}{\partial x_{2}}\left(K_{1}^{*}-\varkappa \mu \frac{\partial \varphi_{1}}{\partial x_{2}}\right)-\frac{\partial}{\partial x_{1}}\left(K_{2}^{*}+\varkappa \mu \frac{\partial \varphi_{1}}{\partial x_{1}}\right)=0, \\
& \frac{\partial K_{1}^{*}}{\partial x_{1}}+\frac{\partial K_{2}^{*}}{\partial x_{2}}+\frac{\partial K_{3}^{*}}{\partial x_{3}}=0 .
\end{aligned}
$$


It follows from system (38) that a function $\mu \frac{\partial \varphi_{2}}{\partial x_{3}}$ exists such that

$$
\begin{gathered}
K_{1}^{*}=\mu \frac{\partial^{2} \varphi_{2}}{\partial x_{1} \partial x_{3}}+\varkappa \mu \frac{\partial \varphi_{1}}{\partial x_{2}}, \quad K_{2}^{*}=\mu \frac{\partial^{2} \varphi_{2}}{\partial x_{2} \partial x_{3}}-\varkappa \mu \frac{\partial \varphi_{1}}{\partial x_{1}} \\
K_{3}^{*}=\mu \frac{\partial^{2} \varphi_{2}}{\partial x_{3}^{2}}
\end{gathered}
$$

with $\Delta \varphi_{2}=0$. . The projection of the equality $\Delta \vec{K}-\frac{\mu}{\eta_{1}} \vec{K}=-\frac{\mu}{\eta_{1}} \vec{K}^{*}$ onto the coordinate axes with (39) in mind will give
a) $\Delta K_{1}-\frac{\mu}{\eta_{1}} K_{1}=-\frac{\mu^{2}}{\eta_{1}} \frac{\partial^{2} \varphi_{2}}{\partial x_{1} \partial x_{3}}-\frac{\varkappa \mu^{2}}{\eta_{1}} \frac{\partial \varphi_{1}}{\partial x_{2}}$,
b) $\Delta K_{2}-\frac{\mu}{\eta_{1}} K_{2}=-\frac{\mu^{2}}{\eta_{1}} \frac{\partial^{2} \varphi_{2}}{\partial x_{2} \partial x_{3}}+\frac{\varkappa \mu^{2}}{\eta_{1}} \frac{\partial \varphi_{1}}{\partial x_{1}}$,
c) $\Delta K_{3}-\frac{\mu}{\eta_{1}} K_{3}=-\frac{\mu^{2}}{\eta_{1}} \frac{\partial^{2} \varphi_{2}}{\partial x_{3}^{2}}$

From (40a) and (40b) we have

$$
\begin{aligned}
& K_{1}=\mu^{2} \eta_{1} \frac{\partial \psi_{1}}{\partial x_{3}}+\mu \frac{\partial^{2} \varphi_{2}}{\partial x_{1} \partial x_{3}}+\varkappa \mu \frac{\partial \varphi_{1}}{\partial x_{2}} \\
& K_{2}=\mu^{2} \eta_{1} \frac{\partial \psi_{2}}{\partial x_{3}}+\mu \frac{\partial^{2} \varphi_{2}}{\partial x_{2} \partial x_{3}}-\varkappa \mu \frac{\partial \varphi_{1}}{\partial x_{1}}
\end{aligned}
$$

where $\Delta \psi_{s}-\frac{\mu}{\eta_{1}} \psi_{s}=0(s=1,2)$ and using the identity $\operatorname{div}(\mu \operatorname{rot} \vec{U})=\frac{\partial K_{1}}{\partial x_{1}}+$ $\frac{\partial K_{2}}{\partial x_{2}}+\frac{\partial K_{3}}{\partial x_{3}}=0$ we can define

$$
K_{3}=-\mu^{2} \eta_{1}\left(\frac{\partial \psi_{1}}{\partial x_{1}}+\frac{\partial \psi_{2}}{\partial x_{2}}\right)+\mu \frac{\partial^{2} \varphi_{2}}{\partial x_{3}^{2}}+f(x, y),
$$

where $f$ is a function resulting from integration of the equality $\operatorname{div}(\mu \operatorname{rot} \vec{U})=0$ with respect to $x_{3}$, which is the solution of boundary value problem (28) if we replace the constant $q$ by the constant $\frac{\mu}{\eta_{1}}$. It can be easily seen that (28) implies $f=0$.

Taking into account (35), (41) and (42) we can obtain the following system

$$
\begin{aligned}
& \frac{\partial u_{1}}{\partial x_{1}}+\frac{\partial u_{2}}{\partial x_{2}}+\frac{\partial u_{3}}{\partial x_{3}}=\frac{\varkappa \mu}{\lambda+2 \mu} \frac{\partial \varphi_{1}}{\partial x_{3}}+\frac{3 \lambda+2 \mu}{\lambda+2 \mu} \gamma T, \\
& \frac{\partial}{\partial x_{2}}\left(u_{3}-\varkappa \varphi_{1}\right)-\frac{\partial}{\partial x_{3}}\left(u_{2}+\mu \eta_{1} \psi_{1}+\frac{\partial \varphi_{2}}{\partial x_{1}}\right)=0, \\
& \frac{\partial}{\partial x_{3}}\left(u_{1}-\mu \eta_{1} \psi_{2}-\frac{\partial \varphi_{2}}{\partial x_{2}}\right)-\frac{\partial}{\partial x_{1}}\left(u_{3}-\varkappa \varphi_{1}\right)=0, \\
& \frac{\partial}{\partial x_{1}}\left(u_{2}+\mu \eta_{1} \psi_{1}+\frac{\partial \varphi_{2}}{\partial x_{1}}\right)-\frac{\partial}{\partial x_{2}}\left(u_{1}-\mu \eta_{1} \psi_{2}-\frac{\partial \varphi_{2}}{\partial x_{2}}\right)=0 .
\end{aligned}
$$


from the equalities $(\lambda+2 \mu) \operatorname{div} \vec{U}-(3 \lambda+2 \mu) \gamma T=D=\varkappa \mu \frac{\partial \varphi_{1}}{\partial x_{3}}$ and $\mu \operatorname{rot} \vec{U}=\vec{K}$.

From (43) we have

$$
\begin{aligned}
u_{1} & =\frac{\partial \widetilde{\varphi}_{3}}{\partial x_{1}}+\mu \eta_{1} \psi_{2}+\frac{\partial \varphi_{2}}{\partial x_{2}}, \\
u_{2} & =\frac{\partial \widetilde{\varphi}_{3}}{\partial x_{2}}-\mu \eta_{1} \psi_{1}-\frac{\partial \varphi_{2}}{\partial x_{1}}, \\
u_{3} & =\frac{\partial \widetilde{\varphi}_{3}}{\partial x_{3}}+\varkappa \varphi_{1} ; \\
\Delta \widetilde{\varphi}_{3}=-2 \frac{\partial \varphi_{1}}{\partial x_{3}} & -\mu \eta_{1}\left(\frac{\partial \psi_{2}}{\partial x_{1}}-\frac{\partial \psi_{1}}{\partial x_{2}}\right)+\frac{3 \lambda+2 \mu}{\lambda+2 \mu} \gamma T .
\end{aligned}
$$

If the change in the temterature $T$ is expressed by means of the function $\widetilde{T}$ according to formula (5) (note that $\Delta \widetilde{T}=0$ ), then (45) will result in

$$
\widetilde{\varphi}_{3}=\varphi_{3}-x_{3} \varphi_{1}-\eta_{1}^{2}\left(\frac{\partial \psi_{2}}{\partial x_{1}}-\frac{\partial \psi_{1}}{\partial x_{2}}\right)+x_{3} \widetilde{T}
$$

Substitution of (46) into (44) shows the validity of Theorem 2.

3. Below we shall give the construction pattern for regular solutions of boundary value problems in asymmetric thermoelasticity. But before we continue our discussion we must define the concept of regular solutions.

In the case of Cosserat's continuum the solution of system (4) defined by seven functions $T, u_{i}, \omega_{i}(i=1,2,3)$ will be called regular if $T$ is twice and $u_{i}, \omega_{i}$ is three times continuously differentiable in the domain $\widetilde{\Omega}$ where $\widetilde{\Omega}$ is the domain $\Omega$ together with the boundaries $x_{1}=x_{1 s}$ and $x_{2}=x_{2 s}(s=0,1)$ and on the surface $x_{3}=x_{3 s}$ they together with their first derivatives can be represented by uniformly converging trigonometric series. It is also assumed that the equilibrium equations are true for $x_{1}=x_{1 s}$ and $x_{2}=x_{2 s}$.

The differential properties imposed on the functions $f_{s 1}, f_{s 2}, \ldots, f_{s 6}$ from (12) are defined by the regularity requirements imposed on the solution.

In the case of Cosserat's pseudo-continuum the solution of (30), which is defined by four functions $T, u_{i}$ is called regular if $T$ is twice and $u_{i}$ is five times continuously differentiable in the domain $\widetilde{\Omega}$ and on the surface $x_{3}=x_{3 s}, T$ together with its first derivative and $u_{i}$ together with its derivatives up to the fourth order can be represented by uniformly converging trigonometric series. We also assume that the equilibrium equations and their first derivatives hold for $x_{1}=x_{1 s}$ and $x_{2}=x_{2 s}$.

The differential properties imposed on the functions $f_{s 1}, f_{s 2}, \ldots, f_{s 5}$ from (35), similar to the case of Cosserat's continuum, are defined by the regularity requirement imposed on the solution. 
4. Let conditions (10b),(11b), (12) be satisfied on the surface of the RP, then

$$
\begin{aligned}
\widetilde{T} & =\sum_{\widetilde{m}=1}^{\infty} \sum_{\widetilde{n}=1}^{\infty} E_{0 m n}\left(x_{3}\right) \sin \left(m x_{1}\right) \sin \left(n x_{2}\right), \\
\varphi_{1} & =\sum_{\widetilde{m}=1}^{\infty} \sum_{\widetilde{n}=1}^{\infty} E_{1 m n}\left(x_{3}\right) \sin \left(m x_{1}\right) \sin \left(n x_{2}\right), \\
\varphi_{2} & =\sum_{\widetilde{m}=0}^{\infty} \sum_{\widetilde{n}=0}^{\infty} E_{2 m n}\left(x_{3}\right) \cos \left(m x_{1}\right) \cos \left(n x_{2}\right), \\
\varphi_{3} & =\sum_{\widetilde{m}=1}^{\infty} \sum_{\widetilde{n}=1}^{\infty} E_{3 m n}\left(x_{3}\right) \sin \left(m x_{1}\right) \sin \left(n x_{2}\right), \\
\psi_{1} & =\sum_{\widetilde{m}=1}^{\infty} \sum_{\widetilde{n}=0}^{\infty} \widetilde{E}_{1 m n}\left(x_{3}\right) \sin \left(m x_{1}\right) \cos \left(n x_{2}\right), \\
\psi_{2} & =\sum_{\widetilde{m}=0}^{\infty} \sum_{\widetilde{n}=1}^{\infty} \widetilde{E}_{2 m n}\left(x_{3}\right) \cos \left(m x_{1}\right) \sin \left(n x_{2}\right), \\
\psi_{3} & =\sum_{\widetilde{m}=0}^{\infty} \sum_{\widetilde{n}=0}^{\infty} \widetilde{E}_{3 m n}\left(x_{3}\right) \cos \left(m x_{1}\right) \cos \left(n x_{2}\right),
\end{aligned}
$$

where $E_{i m n}\left(x_{3}\right)=A_{i m n} \exp \left(-p x_{3}\right)+B_{i m n} \exp \left[p\left(x_{3}-x_{31}\right)\right], i=0,1,2,3, p=$ $\sqrt{m^{2}+n^{2}}, m=\frac{\pi \widetilde{m}}{x_{11}}, n=\frac{\pi \widetilde{n}}{x_{21}}, A_{i m n}$ and $B_{i m n}$ are constant; $\widetilde{E}_{s m n}\left(x_{3}\right)=$ $\widetilde{A}_{s m n} \exp \left(-p_{0} x_{3}\right)+\widetilde{B}_{s m n} \exp \left[p_{0}\left(x_{3}-x_{31}\right)\right], s=1,2, p_{0}=\sqrt{p^{2}+q}, \widetilde{A}_{s m n}$ and $\widetilde{B}_{s m n}$ are constant; $E_{3 m n}\left(x_{3}\right)=\widetilde{A}_{3 m n} \exp \left(-p_{1} x_{3}\right)+\widetilde{B}_{3 m n} \exp \left[p_{1}\left(x_{3}-x_{31}\right)\right]$ with $p_{1}=\sqrt{p^{2}+\frac{4 \sigma_{1}}{\sigma_{2}+2 \sigma_{3}}}, \widetilde{A}_{3 m n}$ and $\widetilde{B}_{3 m n}$ are constant.

Substitution of (47) into (13) and (14) gives

$$
\begin{aligned}
& \omega_{1}=\sum_{\widetilde{m}=1}^{\infty} \sum_{\widetilde{n}=0}^{\infty} \omega_{1 m n}\left(x_{3}\right) \sin \left(m x_{1}\right) \cos \left(n x_{2}\right), \\
& \omega_{2}=\sum_{\widetilde{m}=0}^{\infty} \sum_{\widetilde{n}=1}^{\infty} \omega_{2 m n}\left(x_{3}\right) \cos \left(m x_{1}\right) \sin \left(n x_{2}\right), \\
& \omega_{3}=\sum_{\widetilde{m}=0}^{\infty} \sum_{\widetilde{n}=0}^{\infty} \omega_{3 m n}\left(x_{3}\right) \cos \left(m x_{1}\right) \cos \left(n x_{2}\right), \\
& u_{1}=\sum_{\widetilde{m}=0}^{\infty} \sum_{\widetilde{n}=1}^{\infty} u_{1 m n}\left(x_{3}\right) \cos \left(m x_{1}\right) \sin \left(n x_{2}\right), \\
& u_{2}=\sum_{\widetilde{m}=1}^{\infty} \sum_{\widetilde{n}=0}^{\infty} u_{2 m n}\left(x_{3}\right) \sin \left(m x_{1}\right) \cos \left(n x_{2}\right), \\
& u_{3}=\sum_{\widetilde{m}=1}^{\infty} \sum_{\widetilde{n}=1}^{\infty} u_{3 m n}\left(x_{3}\right) \sin \left(m x_{1}\right) \sin \left(n x_{2}\right),
\end{aligned}
$$

where $\omega_{1 m n}\left(x_{3}\right), \omega_{2 m n}\left(x_{3}\right), \omega_{3 m n}\left(x_{3}\right)$ are known expressions containing the con- 
stants $A_{i m n}, B_{i m n}, \widetilde{A}_{i m n}, \widetilde{B}_{i m n}(i=1,2,3)$ and depend on $x_{3}, m, n$ and depending on $x_{3}, m, n$ and the elastic characteristics of the RP (these expresseions are not given due to their awkwardness).

The functions $\widetilde{T}, \omega_{1}, \omega_{2}, \ldots, u_{3}$ at least formally, satisfy equalibrium equations and boundary conditions (10b) and (11b) (the word "formally" will be omitted as soon as the constants $A_{0 m n}, B_{0 m n}, A_{i m n}, B_{i m n}, \widetilde{A}_{i m n}, \widetilde{B}_{i m n}(i=1,2,3)$ have been defined, convergence of the series and uniqueness of the obtained solution have been proved).

After the right-hand sides of formulas (12) have been substituted by the corresponding trigonometric series and the obtained series have been compared with the series represented by formulas (47a) and (48) we shall have two systems of linear algebraic equations for fixed $m$ and $n$. The first one with a second order amtrix enables one to define the constants $A_{\text {omn }}, B_{0 m n}$, while the second one woth a twelfth-order matrix can be used to define the constants $A_{i m n}, B_{i m n}$, $\widetilde{A}_{i m n}, \widetilde{B}_{i m n}(i=1,2,3)$. Similar to [8], it is proved that the obtained solution or, to be more precise, the series representing the solution, uniformly converge in the domain $\bar{\Omega}=\left\{0 \leq x_{1} \leq x_{11}, 0 \leq x_{2} \leq x_{21}, 0 \leq x_{3} \leq x_{31}\right\}$ and thet the resulted regular solution is unique. In particular, the uniqueness of the solution of boundary value problems of asymmetric elasticity, corresponding to thermoelasticity problems considered in the given paper, as well as of a number of other boundary value ptoblems of asymmetric thermoelasticity is proved in [6]. The solution method can be easily extended to the case of the boundary value and boundary value constant problems of asymmetric thermoelasticity considered in the present work.

Thus a regular solution of boundary value problem (4), (10b), (11b), (12) has been obtained for $g_{s 1}=0, g_{s 2}=0, \ldots, g_{s 7}=0$ and $\widetilde{g}_{s 1}=1, \widetilde{g}_{s 2}=1, \ldots, \widetilde{g}_{s 7}=1$.

We should also say a few words about the above-mentioned second- and twelfth-order matrices or, to be more exact, of the determinants $\Delta_{2}$ and $\Delta_{12}$ of these matrices. We shall start with $\Delta_{12}$. Scaling in the expressions for $E_{i m n}$ and $\widetilde{E}_{i m n}(i=1,2,3)$ can be always performed so the the elements of the twelfthorder matrix should be bounded values for any $\widetilde{m}$ and $\widetilde{n}$ (including the case when $\widetilde{m} \rightarrow \infty, \widetilde{n}=\widetilde{n}_{0}=$ const or $\widetilde{n} \rightarrow \infty, \widetilde{m}=\widetilde{m}_{0}=$ const, or when both $\widetilde{m} \rightarrow \infty$ and $\widetilde{n} \rightarrow \infty)$. If this is the case, then analizing the system for $\widetilde{m} \rightarrow \infty$, $\widetilde{n}=\widetilde{n}_{0}$, for $\widetilde{n} \rightarrow \infty, \widetilde{m}=\widetilde{m}_{0}$ and for $\widetilde{m} \rightarrow \infty, \widetilde{n} \rightarrow \infty$, one can easily see that for all those cases $\Delta_{12} \neq 0$, while when $\widetilde{m}=\widetilde{m}_{0}$ and $\widetilde{n}=\widetilde{n}_{0}$ by virtue of the uniqueness theorem we have $\Delta_{12} \neq 0$. The same holds for $\Delta_{2}$ too, though the expression for this detertminant can be immediately written out and analysed.

Quite similarly one can find and analyzed a solution of any other problem from the class of boundary value problems (4), (10), (11), (12) with the only difference that different boundary conditions for $x_{1}=x_{1 s}$ and $x_{2}=x_{2 s}$ in the expressions for $\widetilde{T}, \varphi_{1}, \varphi_{2}, \ldots, \psi_{3}$ (see formulas (47)) will correspond to different trigonometrical functions.

If in (47) we omit the last equality (the expression for $\psi_{3}$ ) and in the expression for $p_{0}$ we replace $q$ by $\frac{\mu}{\eta_{1}}$, then the functions $\widetilde{T}, \varphi_{1}, \varphi_{2}, \varphi_{3}, \psi_{1}, \psi_{2}$ 
will define, according to Cosserat's pseudo-continuum, the solution of boundary value problem (30), (33b), (34b), (35). The comments and generalizations given to boundary value problem (4), (10b), (11b) and (12) for Cosserat's continuum, exactly hold for the same problem in CosseratTs pseudo-continuum, i.e. problem (30), (33), (35b), (35). The only difference is that unlike in the case of Cosserat's continuum, where one has to solve systems of linear equations with a matrices of the second and twelfth order, in the case of Cosserat's pseudo-continuum we have a second-order and tenth-order matrices.

5. According to asymmetric theory, consider a RP multilayer along $x_{3}$ (or MRP in the abbreviated form) occupying the domain $\Omega_{3} . \Omega_{3}$ is a union of the domains $\Omega_{31}=\left\{0<x_{1}<x_{11}, 0<x_{2}<x_{21}, 0<x_{3}<x_{31}\right\}, \Omega_{32}=\{0<$ $\left.x_{1}<x_{11}, 0<x_{2}<x_{21}, x_{31}<x_{3}<x_{32}\right\}, \ldots, \Omega_{3 \beta}=\left\{0<x_{1}<x_{11}, 0<\right.$ $\left.x_{2}<x_{21}, x_{3(\beta-1)}<x_{3}<x_{3 \beta}\right\}$, contacting along the planes $x_{3}=x_{3 j}$, where $j=1,2, \ldots, \beta-1$, and $\beta$ is the number of layers. Each layer has its elastic and thermal characteristics. With $x_{1}=0$ and $x_{1}=x_{11}$ conditions (10) are simultaneously satisfied for all layers while with $x_{2}=0$ and $x_{2}=x_{21}$ conditions (11) hold.

If the body occupies the domain $\Omega_{3}$, then conditions (10), (11), (12) are satisfied on its faces with $x_{31}$ replaced by $x_{3 \beta}$ in (12). On the contact planes $x_{3}=x_{3 j}\left(x_{3}=x_{3 j}\right.$ is the contact plane of the $j$-th layer contacting the $\mathrm{j}+1$-th layer) the following conditions

$$
\begin{array}{ll}
T_{j}-T_{j+1}=\tau_{j 1}\left(x_{1}, x_{2}\right), & \lambda_{j}^{*} \frac{\partial T_{j}}{\partial x_{3}}-\lambda_{j+1}^{*} \frac{\partial T_{j+1}}{\partial x_{3}}=\tau_{j 2}\left(x_{1}, x_{2}\right), \\
u_{1 j}-u_{1(j+1)}=q_{j 1}\left(x_{1}, x_{2}\right), & N_{31 j}-N_{31(j+1)}=Q_{j 1}\left(x_{1}, x_{2}\right), \\
u_{2 j}-u_{2(j+1)}=q_{j 2}\left(x_{1}, x_{2}\right), & N_{32 j}-N_{32(j+1)}=Q_{j 2}\left(x_{1}, x_{2}\right), \\
u_{3 j}-u_{3(j+1)}=q_{j 3}\left(x_{1}, x_{2}\right), & N_{33 j}-N_{33(j+1)}=Q_{j 3}\left(x_{1}, x_{2}\right), \\
\omega_{1 j}-\omega_{1(j+1)}=q_{j 4}\left(x_{1}, x_{2}\right), & M_{31 j}-M_{31(j+1)}=Q_{j 4}\left(x_{1}, x_{2}\right), \\
\omega_{2 j}-\omega_{2(j+1)}=q_{j 5}\left(x_{1}, x_{2}\right), & M_{32 j}-M_{32(j+1)}=Q_{j 5}\left(x_{1}, x_{2}\right), \\
\omega_{3 j}-\omega_{3(j+1)}=q_{j 6}\left(x_{1}, x_{2}\right), & M_{33 j}-M_{33(j+1)}=Q_{j 6}\left(x_{1}, x_{2}\right)
\end{array}
$$

or

$$
\begin{array}{ll}
T_{j}-T_{j+1}=\tau_{j 1}\left(x_{1}, x_{2}\right), & \lambda_{j}^{*} \frac{\partial T_{j}}{\partial x_{3}}-\lambda_{j+1}^{*} \frac{\partial T_{j+1}}{\partial x_{3}}=\tau_{j 2}\left(x_{1}, x_{2}\right), \\
N_{31 j}=Q_{j 1}\left(x_{1}, x_{2}\right), & N_{31(j+1)}=\widetilde{Q}_{j 1}\left(x_{1}, x_{2}\right), \\
N_{32 j}=Q_{j 2}\left(x_{1}, x_{2}\right), & N_{32(j+1)}=\widetilde{Q}_{j 2}\left(x_{1}, x_{2}\right), \\
u_{3 j}-u_{3(j+1)}=q_{j 3}\left(x_{1}, x_{2}\right), & N_{33 j}-N_{33(j+1)}=Q_{j 3}\left(x_{1}, x_{2}\right), \\
\omega_{1 j}-\omega_{1(j+1)}=q_{j 4}\left(x_{1}, x_{2}\right), & M_{31 j}-M_{31(j+1)}=Q_{j 4}\left(x_{1}, x_{2}\right), \\
\omega_{2 j}-\omega_{2(j+1)}=q_{j 5}\left(x_{1}, x_{2}\right), & M_{32 j}-M_{32(j+1)}=Q_{j 5}\left(x_{1}, x_{2}\right), \\
M_{33 j}=Q_{j 6}\left(x_{1}, x_{2}\right), & M_{33(j+1)}=\widetilde{Q}_{j 6}\left(x_{1}, x_{2}\right)
\end{array}
$$

are defined. The right-hand sides of equalities (49) and (50) are defined functions and $\lambda_{j}^{*}$ and $\lambda_{j+1}^{*}$ are thermal conductivity coefficients.

When we state the problem of thermoelastic equilibrium of a MRP we give expressions for the functions $\widetilde{T}^{(j)}, \varphi_{1}^{(j)}, \varphi_{2}^{(j)}, \varphi_{3}^{(j)}, \psi_{1}^{(j)}, \psi_{2}^{(j)}, \psi_{3}^{(j)}$ for the $j$-th layer bearing in mind conditions (10), (11), and following the technique of 
the previous section construct a system of $2 \beta$ equations in $2 \beta$ unknowns and another system of $12 \beta$ equations in $12 \beta$ unknowns. Solvability of the system, convergence of the corresponding series and uniqueness of the obtained regular solutions of the respective boundary contact problems of thermoelasticity are proved (the solution for the MRP is called regular if each of the solutions $u_{1 j}$, $u_{2 j}, u_{3 j}$ is regular).

Besides the given contact conditions, a number of other contact conditions can be constructed under which the solution of boundary contact problems of thermoelasticity can be likewise effective.

Solutions of boundary contact problems for Cosserat's pseudo-continuum are considered in a similar way.

In conclusion, we should note that the constructed effective solutions of boundary vale and boundary contact problems, as a rule, become elementary. Indeed, if the functions defined on the surface $x=x_{3 s}$ (although we imply boundary value problems the above-stated can be easily extended to boundary contact problems as well) can be represented by finite trigonometric series, then the solutions can be also represented by finite series the summands of which are elementary functions. In particular, this statement can be used to specify and define new elasticity characteristics of a material $\sigma_{1}, \sigma_{2}, \sigma_{3}, \sigma_{4}$ and $\eta_{1}, \eta_{2}$, naturally, using high-quality measuring instruments.

\section{REFERENCES}

1. E. Cosserat and F. Cosserat, Theórie des corps déformables. Hermann, Paris, 1909.

2. C. A. Truesdell and R. A. Toupin, The classical field theories. Handbuch der Physik, Band 3, Teil 1, Springer, Berlin, 1960.

3. W. Nowacki, Theory of elasticity. (Translated from Polish into Russian) Mir, Moscow, 1975.

4. A. C. Eringer, Theory of micropolar elasticity. J. Math. Mech. 15(1966), 909-923.

5. W. Nowacki, Couple stresses in the theory of thermoelasticity. Bull. Acad. Polon. Sér. Sci. Techn. 14(1966), No. 8.

6. V. D. Kupradze, T. G. Gegelia, M. O. Basheleishvili, and T. V. Burchuladze, Three-dimensional problems of the mathematical theory of elasticity and thermoelasticity. (Translated from Russian) North-Holland Series in Applied Mathematics and Mechanics, v. 25, North-Holland Publ. Co., Amsterdam-New York-Oxford, 1979; Russian original: Nauka, Moscow, 1976.

7. B. Carbonaro and R. Russo, On some classical theorems in micropolar elasticity. Internat. J. Engrg. Sci. 23(1985), 119-129.

8. N. Khomasuridze, Thermoelastic equilibrium of bodies in generalized cylindrical coordinates. Georgian Math. J. 5(1998), No. 6, 521-544.

(Received 18.09.2000; revised 22.09.2001)

Author's address:

I. Vekua Institute of Applied Mathematics

I. Javakhishvili Tbilisi State University

2, University St., Tbilisi 380043

Georgia 